\title{
Características poblacionales y factores asociados a embarazo en mujeres adolescentes de Tuluá, Colombia
}

\author{
Yessica Alexandra Sánchez Valencia ${ }^{1}$, Luis Alfonso Mendoza Tascón ${ }^{1,2}$, Mónica \\ Beatriz Grisales López ${ }^{1}$, Leidy Yohanna Ceballos Molina ${ }^{1}$, Johanna Catalina \\ Bustamente Farfan ${ }^{1}$, Edgar Muriel Castañeda ${ }^{1}$, Luis Alberto Chaverra ${ }^{1}$, Maria \\ Eulalia Acuña Polo'.
}

${ }^{1}$ Facultad de Ciencias de la Salud, Unidad Central del Valle. ${ }^{2}$ Fundación Hospital San José de Buga, Colombia.

\section{RESUMEN}

Antecedentes: El embarazo en adolescentes es un problema social, con múltiples determinantes asociados. Colombia tiene una tasa elevada de embarazo adolescente. Objetivo: Determinar los factores de riesgo asociados a embarazo en mujeres adolescentes. Métodos: Estudio de casos y controles, con 151 mujeres adolescentes de 19 años o menos, embarazadas, con hijos o antecedente de embarazo y 302 adolescentes controles con actividad sexual (AS) sin historia de embarazo. Las asociaciones se determinaron mediante Odds Ratio. Para el análisis multivariado se empleó regresión logística $(R L)$. Con las variables de la $R L$ se creó un modelo predictor de embarazo, evaluándose su rendimiento mediante sensibilidad, especificidad y área bajo ROC. Resultados: Hubo asociación significativa con embarazo las variables individuales: no encontrarse escolarizadas o estudiando, inicio de AS a los 15 o menos años y no usar métodos de planificación familiar (PF) en todas las relaciones sexuales; en variables familiares: escolaridad materna menor que secundaria, no educación sexual del adolescente por la madre y no suministro de métodos de PF por la madre, y en las variables socio-culturales: pertenecer al estrato socio-económico 1 y no haber recibido educación en PF por parte de personal docente, de salud $u$ otro diferente a los padres. El modelo tuvo sensibilidad $92,1 \%$, especificidad $68,9 \%$ y área bajo ROC 0,805. Conclusiones: Las causas asociadas a embarazo involucran factores individuales, familiares y socio-culturales. La promoción de la abstinencia es un objetivo válido, especialmente entre adolescentes más jóvenes, sin embargo, la evidencia científica mundial demuestra que no es suficiente para evitar embarazos no deseados.

PALABRAS CLAVE. Embarazo adolescente, factores de riesgo, anticoncepción, educación sexual

\section{SUMMARY}

Background: Teenage pregnancy is a social problem associated with multiple determinants. Colombia has a high rate of teenage pregnancy Objective: To determine risk factors associated with teenage pregnancy in women and develop a predictive scale pregnancy in adolescent girls. Methods: Case-control study with 151 female adolescents aged 19 or younger, pregnant, with children or a history of pregnancy and 302 controls sexually active female adolescents (SA) with no history of pregnancy. Associations were determined by odds ratio (OR). Multivariate analysis was used logistic regression (LR). With the LR variable predictor of pregnancy model was created, evaluating their performance by sensitivity, specificity and area under ROC. Results: Pregnancy was significantly associated with individual variables: not be educated or studying, SA start at 15 or younger and not use methods of family planning (FP) in all sexual intercourse; family variables: 
maternal education less than high school, adolescent sex education for the mother and FP methods supply by the mother, and socio-cultural variables: belong to the socio-economic stratum 1 and have not received FP education by teachers, health or different to parents. The model had $92.1 \%$ sensitivity, specificity $68.9 \%$ and area under ROC 0.805. Conclusions: The causes related to pregnancy involve individual, family and socio-cultural factors. Promoting abstinence is a worthwhile goal, especially among younger adolescent, however, the global scientific evidence shows that it is not enough to prevent unwanted pregnancies.

\section{KEY WORDS. Adolescent pregnancy, risk factors, contraception, sexual education}

\section{INTRODUCCIÓN}

La salud sexual y reproductiva (SSR) es un componente central en la vida de adolescentes y jóvenes. Las decisiones y conductas que tomen las y los adolescentes en estos temas, serán diferentes según las oportunidades económicas, laborales, educativas que han tenido, la oferta de anticoncepción, la situación socioeconómica, las características personales y las normas sociales y la influencia del medio o contexto social en que viven y crecen (1). En las últimas décadas, el embarazo adolescente se ha convertido en un importante problema de salud $(2,3)$, y es considerado una problemática social cuyo efecto se traduce en falta de oportunidades y aplanamiento de las perspectivas futuras de vida de la adolescente embarazada, su hijo/a y su familia. Las adolescentes embarazadas tienen menos probabilidades que los adultos de ser capaz de obtener un permiso legal y seguro para abortar en países donde el aborto está autorizado legalmente. También son menos propensos que los adultos a obtener atención prenatal, parto y postnatal calificado (4).

Cerca de 16 millones de adolescentes entre 15 y 19 años tienen un hijo cada año. La literatura nos informa que $62,8 \%$ de los adolescentes en escuelas norteamericanas son sexualmente activos y cerca de 850.000 adolescentes resultan embarazadas cada año. En Estados Unidos de Norteamérica (EEUU) en 2004, se estimó que $41,2 \%$ de embarazos ocurren en adolescentes de 15 a 19 años y $20 \%$ de estos, terminan en abortos inducidos, sin embargo, en los últimos años han logrado reducir las tasas de embarazos en un 33\%, asociado a la reducción de tasas de actividad sexual (AS) y aumento del empleo más regular y prolongado de métodos anticonceptivos (5).

Los hijos de madres adolescentes representan aproximadamente el $11 \%$ de todos los nacimientos en el mundo, con un $95 \%$ de ellas en países en desarrollo. Colombia tiene una de las mayores tasas de embarazo adolescente de América Latina, donde 1 de cada 5 adolescentes entre 15 y 19 años ha estado embarazada, lo cual significa un obstáculo para el desarrollo individual y social del país (6); esta situación es más grave en víctimas del desplazamiento, donde $63 \%$ de las cuales han estado o están embarazadas de su primer hijo antes de cumplir los 19 años (7). En Ecuador, 2 de cada 3 adolescentes de 15 a 19 años sin educación, son madres o están embarazadas por primera vez; es así que en la última década la tendencia del incremento del embarazo en adolescentes menores de 15 años es de $74 \%$ y en mayores de 15 años de $9 \%$, siendo la tendencia de la fecundidad adolescente en Ecuador la más alta de la Región Andina, llegando a 100 por 1000 nacidos vivos (nv) (8). Para Bolivia, cerca de un $18 \%$ de las adolescentes de 15 a 19 años ya son madres o están embarazadas; al menos la mitad de estos embarazos no fueron planificados al momento de la concepción (9). Para Chile en el año 2009, el número total de nacidos vivos fue de 252.240 y de estos, el $16,14 \%$ correspondió a nacidos vivos de madres adolescentes; al desagregar por grupo etario, 15,7\% corresponden a adolescentes de $15-19$ años y $0,4 \%$ restante a adolescentes de 10-14 años (10).

El embarazo adolescente se ha vinculado a múltiples determinantes sociales (bajo nivel socioeconómico, bajo nivel de escolaridad, ausencia de proyecto de vida, así como también se le ha relacionado con inequidades en el acceso a servicios de SSR), políticos (insuficiente educación sexual en el sistema educativo en muchos de los países, falta de políticas públicas de SSR para adolescentes y mujeres jóvenes no unidas, y la no consideración de los derechos sexuales y reproductivos de las adolescentes), individuales (inicio cada vez más temprano del ejercicio de la actividad sexual y bajas tasas de planificación familiar) y de los medios de comunicación ("erotización" de los medios de comunicación).

El objetivo de esta comunicación es determinar los factores de riesgo asociados al embarazo en mujeres adolescentes, desarrollar una escala predictiva de embarazo en mujeres adolescentes de la cuidad de Tuluá, Colombia, y suministrar información para la creación de políticas públicas de salud sexual y reproductiva en adolescentes. 


\section{PACIENTES Y MÉTODOS}

Información recolectada como parte de un estudio de casos y controles, de los factores asociados al embarazo en mujeres adolescentes con edades igual o menor a 19 años, asistentes a los servicios de salud de dos instituciones prestadoras de servicios de salud (IPS), de la ciudad de Tuluá, Colombia en el 2012.

Para los casos, se incluyeron mujeres adolescentes de 19 años o menos años, en embarazo, con aborto espontáneo o provocado, post-parto inmediato o con hijo (s), escolarizadas o no, asistiendo a Servicios de Salud Amigables para Adolescentes y Jóvenes, programas de control prenatal, planificación familiar y control de crecimiento y desarrollo de su/s hijo/s en los Hospitales Rubén Cruz y Tomás Uribe, Tuluá, Colombia. Se excluyeron como casos, adolescentes con resultados de pruebas de embarazo falsos positivos, que no pertenecieran al área urbana de la ciudad, que padecieran alguna discapacidad o incapacidad para dar la información para esta investigación y que no firmaran el consentimiento informado. Para los controles, se incluyeron mujeres adolescentes con 19 años o menos de edad, que hayan iniciado la actividad sexual, escolarizadas o no, sin historia de embarazos previos, que asistieran a servicios o programas de salud diferentes a control prenatal, como Servicios de Salud Amigables para Adolescentes y Jóvenes, detección de alteración del joven, planificación familiar, servicios odontológicos, vacunación contra el virus del papiloma humano (VPH) y a consultas espontáneas de los Hospitales Ruben Cruz y Tomas Uribe, Tuluá, Colombia. Se excluyeron como controles adolescentes que no pertenecieran al área urbana de la ciudad, que padecieran alguna discapacidad o incapacidad para dar la información para esta investigación y que no firmaran el consentimiento informado

La variable de resultado fue ser mujer adolescente de 19 años o menos de edad, gestante, o con historia de aborto espontáneo o provocado, en post-parto inmediato o con hijo/s. Las variables independientes incluyeron datos de la adolescente como edad, estrato socioeconómico, barrio donde vive, ocupación, escolaridad, persona con quien vive, tipo de seguridad social en salud, edad de la menarca, edad de la primera relación sexual, número de embarazos, progenitor del hijo/s, edad del padre del hijo/s, embarazo planeado, causas consideradas del embarazo, pensamiento o intención de interrumpir el embarazo, número de hijos, historia de planificación familiar, frecuencia de uso de métodos de planificación familiar, frecuencia de uso de condón masculino de su pareja/s, edad de inicio de la planificación familiar, tipos de métodos de planificación familiar usados, planificación familiar al momento del embarazo, tipo de planificación familiar al momento del embarazo, dependencia económica al momento del embarazo, número de personas con las que habita, educación sexual por padres y personas diferentes a los padres como educadores y personal de salud, educación en planificación familiar por los padres y personas diferentes a los padres como educadores y personal de salud, creencias sobre los mejores métodos de evitar un embarazo, uso del tiempo libre, creencias sobre los riesgos del embarazo para las adolescentes y sus hijos, primera relación sexual bajo efectos de alcohol o drogas, permisividad sexual del adolescente o de sus pares o amigos hacia la actividad sexual en la adolescencia, número de hermanos, lugar que ocupa entre los hermanos, edad del hermano mayor, hermanos que en la adolescencia hayan sido padre o madre. Datos de la madre y padre como edad, ocupación, escolaridad, educación de la madre y padre sobre sexualidad a sus hijas, educación de la madre y padre sobre planificación familiar a sus hijas, si es la madre o el padre quien consigue métodos de planificación familiar a sus hijas.

Los datos fueron obtenidos mediante una encuesta estructurada por entrevista personal de manera individual bajo la supervisión y asesoría de un investigador. Esta encuesta cumplió con los requisitos de confiabilidad, validez y objetividad y fue aplicada en una prueba piloto a un grupo de adolescentes de las mismas instituciones de atención en salud donde se realizó el trabajo.

Para evitar sesgos de información, a las adolescentes del estudio de cada institución de salud se explicó los alcances y objetivos de la investigación, sus procedimientos y los resultados que se esperaban. Igualmente, se les indicó los beneficios que traería para ellos, las familias y la comunidad. A su vez, se les aseguró preservar la identidad y reserva de toda la información suministrada. Las encuestas eran anónimas, y se manejaron códigos que sólo la encuestada conocía y registrados bajo custodia.

Para evitar sesgos de selección, se tuvo en cuenta el estricto cumplimiento de los criterios de inclusión y exclusión. Los controles se extrajeron mediante muestreo aleatorio sistemático, a partir de los registros de los Servicios de Salud Amigables para Adolescentes y Jóvenes y otros programas de las dos instituciones, que cumplieran con los criterios de inclusión.

El tamaño de la muestra se obtuvo mediante el Programa estadístico Epidat 3.1. La muestra con una relación de casos:controles de 1:2, fue de 151 casos y 302 controles, lo cual nos permite determinar un OR de 1,9, con un nivel de confianza de $95 \%$ y poder estadístico de $80 \%$.

Los datos fueron importados desde Microsoft Office Excel 2007 al paquete estadístico Stata ${ }^{\circledR}$ 11.0 donde fueron procesados. Las variables continuas se describieron de acuerdo con su distribución con promedios y desviación estándar o medianas 
y rangos intercuartiles. Para comparar diferencias se empleó la prueba t-test no pareado o prueba de Mann-Whitney según correspondiera. Las variables categóricas se expresaron en frecuencias y proporciones y fueron comparadas usando la prueba de Chi2 de Pearson. Algunas variables se agruparon para su análisis. Para determinar la asociación entre cada variable independiente y la variable resultado, se calculó el OR con su intervalo de confianza del 95\% (IC 95\%) (11). Para el análisis y determinación de las asociaciones, las variables se dividieron en tres niveles: nivel individual, nivel familiar y nivel sociocultural de los adolescentes y sus padres. El análisis multivariado mediante regresión logística $(R L)$ fue realizado para cada uno de los tres niveles. En la $R L$ se incluyeron las variables que en el bivariado hubieran mostrado significancia estadística, con probabilidad de retiro si la $p>0,05$ (12). Las variables con significancia estadística fueron determinadas por el método "Backward:Wald", como lo describe Maroco (13). Finalmente, teniendo en cuenta los coeficientes de RL de cada una de las variables, en un modelo que incluyera a todas las variables con significativa asociación, se establecieron criterios mayores y menores, según los puntajes de los coeficientes de RL, definiendo como criterio mayor aquella/s variable/s con un valor de coeficiente de regresión logística de dos veces superior (o cercano a dos veces superior) al valor de coeficiente de $R L$ de las demás variables, a partir de los cuales se creó la escala predictiva. Se considero un valor de $\mathrm{p}<0,05$ como significativo. El trabajo fue aprobado por el Comité de Ética de la Unidad Central del Valle y el Secretario de Educación Municipal de Tuluá, Colombia. Los Rectores de los colegios de la ciudad, dieron su consentimiento para la realización de esta investigación.

\section{RESULTADOS}

Un total de 151 casos y de 302 controles fueron incluidos en el estudio. La mediana de edad fue 17 años (RI: 16-18 años), sin hallar diferencias estadísticamente significativas entre casos y controles. Hallamos una menor escolaridad entre las mujeres casos (mediana 9 años de estudio; RI: 7-11 años) que entre las controles (mediana 10 años de estudio; RI: 9-11 años) $(p<0,001)$. En cuanto a la ocupación de las adolescentes, encontramos que entre los casos $50 \%$ (n: 75) eran amas de casa, 35,8\% (n: 54) se encontraban en el colegio y entre los controles $84,1 \%$ (n: 254) se encontraba estudiando y $10,6 \%$ (n: 32) eran amas de casa $(p<0,001)$. El 93,3\% de las adolescentes caso vs el 76,8\% de los controles tenían al momento del estudio seguridad social en salud $(p<0,001)$. La mayoría de la adolescentes caso dependían del conyugue ( $\mathrm{n}: 96 ; 63,6 \%$ ), mientras que la adolescentes control dependían primordialmente de los padres ( $n: 249 ; 82,5 \%)(p<0,001)$. Aunque las medianas de la edad de inicio de la actividad sexual fue de 15 años para ambos grupos, las mujeres caso la empezaron en mayor proporción ( $\mathrm{n}$ : 118; 78,2\%) que los controles (n: 188; 62,3\%) (Tabla I).

En cuanto a planificación familiar, encontramos que las adolescentes casos, en menor proporción habían planificado ( $\mathrm{n}$ : $100 ; 66,2 \%$ vs n: 242; $80,1 \%, p<0,01)$, sin diferencia en la edad de inicio de la planificación familiar (15,5 años $\pm 1,4$ años). En cuanto al uso de métodos de planificación familiar en todas las relaciones sexuales, 138 $(45,7 \%)$ adolescentes control respondieron afirmativamente, mientras esto ocurrió en $32(21,2 \%)$ de las adolescentes caso ( $p<0,001)$. El 62,5\% (n: 283) de todas las adolescentes del estudio no usaban en todas sus relaciones métodos de planificación familiar y entre estas, el 17\% (n: 77) nunca habían usado ningún método de planificación familiar (Tabla I). En cuanto al uso del condón masculino, 21 $(13,9 \%)$ adolescentes caso y 103 (34,1\%) adolescentes control, afirmaron que sus parejas sexuales lo usaban siempre $(p<0,001)$. De igual manera, 130 $(86,1 \%)$ casos y $199(65,9 \%)$ controles, admitieron usarlo a veces o nunca durante sus relaciones sexuales $(p<0,001)$. Para las adolescentes caso los mejores métodos para evitar un embarazo en la adolescencia son la educación en planificación familiar $(72,2 \%)$ y la educación sexual $(17,2 \%)$, mientras que para las adolescentes control fueron la planificación familiar $(57,9 \%)$ y educación sexual $(34,8 \%)(p=0,003)$ (Tabla I).

En cuanto a los problemas secundarios que se derivan de un embarazo en la adolescencia, para las adolescentes caso las preocupaciones fueron los problemas de salud (53,6\%), ausentismo escolar o retiro de sus estudios $(10,6 \%)$ y problemas familiares $(9,3 \%)$, mientras para las adolescentes control los principales fueron los problemas económicos $(29,1 \%)$, de salud $(13,9 \%)$ y emocionales (7,9\%) (Tabla I).

Encontramos que $352(77,7 \%)$ de las mujeres adolescentes de este estudio afirmaron aprobar el inicio y mantenimiento de la actividad sexual en la adolescencia, sin hallar diferencia entre los dos grupos (Tabla I).

Entre el grupo de adolescentes casos, encontramos que $14,6 \%$ eran madres de 2 o más hijos. En el $58,3 \%$ de los casos el padre del hijo era el conyugue, $35 \%$ el novio y $6 \%$ otra persona. En el $29,8 \%$ de los casos el padre del hijo fue adolescente, mientras $70,2 \%$ fue mayor de 19 años, siendo $21,9 \%$ adultos (Tabla II). De la totalidad de adolescentes gestantes o con hijos, 89 (58,9\%), admitieron que los embarazos no fueron planeados, y las razones argumentadas por las propias adolescentes fueron la no planificación familiar (n: 48; 53,9\%) y uso inadecuado de los métodos de planificación familiar (n: 35; 39,3\%), entre otras causas. El 80,8\% (n: 122) no se encontraba planificando al momento del embarazo, mientras $7,9 \%(n: 12)$ pensó en interrumpir el embarazo (Tabla II). 
Tabla I

CARACTERÍSTICAS INDIVIDUALES

\begin{tabular}{l} 
Variable individual \\
\hline Edad (años) \\
Ocupación al momento de la encuesta
\end{tabular}

Con Seguridad Social en salud

De quien depende económicamente la adolescente

$$
\begin{array}{r}
\text { Conyugue } \\
\text { Padres } \\
\text { Abuelos } \\
\text { Otro familiar } \\
\text { Ella misma }
\end{array}
$$

Edad de menarca

Otro

Edad de primera relación sexual

Inicio de la actividad sexual entre los 9 y 15 años de edad

Ha planificado alguna vez

Edad en que inició la PF

Mejor opción para prevenir el embarazo en la adolescencia

Educación sexual

Educación en PF

Retrasar el inicio de la AS

Fomentar las actividades lúdicas

Uso de métodos de PF en todas sus relaciones sexuales

$$
\begin{array}{r}
\text { Siempre } \\
\text { A veces } \\
\text { Nunca }
\end{array}
$$

Frecuencia de uso de condón en sus relaciones sexuales

$$
\begin{array}{r}
\text { Siempre } \\
\text { A veces } \\
\text { Nunca }
\end{array}
$$

Riesgos del embarazo en adolecentes para la madre y el

hijo

Problemas que traería el embarazo en adolecentes

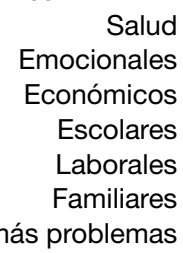

La adolecente aprueba o desaprueba las relaciones sexuales a su edad

\section{Control}

(n: 302)

Caso

17 (Rl: 16-17)

n: 302

254 (84,1\%)

$6(2 \%)$

$32(10,6 \%)$

$10(3,3 \%)$

10 (RI: 9-11)

232 (76,8\%)

$$
\begin{array}{r}
36(11,9 \%) \\
249(82,5 \%) \\
4(1,3 \%) \\
6(2 \%) \\
6(2 \%) \\
1(0,3 \%)
\end{array}
$$

$12,2( \pm 1,4)$

15 (RI: 14-16)

188 (62,3\%)

242 (80,1\%)

$15,5( \pm 1,3)$

105 (34,8\%)

$175(57,9 \%)$

$17(5,6 \%)$

$5(1,7 \%)$

$138(45,7 \%)$

$118(39,1 \%)$

$46(15,2 \%)$

103 (34,1\%)

$132(43,7 \%)$

67 (22,2\%)

264 (87,4\%)

$$
\begin{array}{r}
42(13,9 \%) \\
24(7,9 \%) \\
88(29,1 \%) \\
15(5 \%) \\
13(4,3 \%) \\
9(3 \%) \\
111(36,8 \%) \\
236(78,1 \%)
\end{array}
$$

(n: 151)

17 (RI: 16-18)

0,1512

n: 151

54 (35,8\%)

$12(7,9 \%)$

$75(49,7 \%)$

$10(6,6 \%)$

9 (RI: 7-11)

$141(93,3 \%)$

$<0,001$

$<0,001$

$<0,001$

$96(63,6 \%)$

$31(20,5 \%)$

$2(1,3 \%)$

$13(8,6 \%)$

$4(2,6 \%)$

$5(3,3 \%)$

$12,2( \pm 1,4)$

0,783

15 (RI: 14-15)

$<0,01$

$118(72,2 \%)$

0,001

$100(66,2 \%)$

0,023

$15,3( \pm 1,6)$

0,11

0,003

$26(17,2 \%)$

$109(72,2 \%)$

$14(9,3 \%)$

2 (1,3\%)

$32(21,2 \%)$

$88(58,3 \%)$

$31(20,5 \%)$

$21(13,9 \%)$

$90(59,6 \%)$

$40(26,5 \%)$

107 (70,9\%)

$81(53,6 \%)$

$11(7,3 \%)$

$7(4,6 \%)$

$16(10,6 \%)$

$2(1,3 \%)$

$14(9,3 \%)$

$20(13,2 \%)$

$115(76,2 \%)$
$<0,001$

$<0,001$

$<0,001$

$<0,01$

0,673

PF: Planificación familiar 
Tabla II

CARACTERÍSTICAS INDIVIDUALES DE LAS ADOLESCENTES EMBARAZADAS

\begin{tabular}{cc}
\hline Variable individual & Caso \\
& $(\mathrm{n}: 151)$ \\
\hline
\end{tabular}

Número de embarazos incluido el actual

\section{1}

3

$$
\begin{array}{r}
129(85,4 \%) \\
20(13,3 \%) \\
2(1,3 \%)
\end{array}
$$

Padre del hijo de la adolescente Conyugue: esposo o marido Novio Otro

No responde

Edad del padre del hijo de la adolescente

Edad del padre del hijo de la adolescente

15-19 años
$20-25$ años
$26-40$ años

Embarazo planeado

Si no fue planeado porque ocurrió el embarazo

No uso de métodos de PF Uso inadecuado de métodos de PF Desconocimiento de métodos de PF o carencia para adquirirlos Curiosidad

Pensó en interrumpir el embarazo actual

Se encontraba planificando al momento en que se embarazo

Método de PF empleado en el momento del embarazo

$$
\begin{array}{r}
\text { Condón } \\
\text { Anticonceptivo inyectable } \\
\text { Anticonceptivo oral }
\end{array}
$$
Otro

PF: Planificación familiar

Encontramos que $72(47,7 \%)$ de las adolescentes caso y $249(82,5 \%)$ de adolescentes control vivían con sus padres ( $p<0,001)$. El 38,3\% ( $n$ : 54$)$ de casos y $16,3 \%(n: 25)$ de controles, tenían más de tres hermanos $(p<0,001)$, sin diferencia entre los dos grupos del lugar que la adolescente ocupaba entre los hermanos (Tabla III).
$88(58,3 \%)$

$53(35 \%)$

$3(2 \%)$

$6(4 \%)$

22 (RI: 19-25)

$45(29,8 \%)$

$73(48,3 \%)$

$33(21,9 \%)$

$62(41 \%)$

$48(53,9 \%)$

$35(39,3 \%)$

$5(5,6 \%)$

$1(1,1 \%)$

$12(7,9 \%)$

$29(19,2 \%)$

$15(51,7 \%)$

$10(34,5 \%)$

$3(10,3 \%)$

$1(3,5 \%)$
Hubo un mayor número de madres ama de casa entre las adolescentes caso $(66,2 \%$ vs $56 \%$; $\mathrm{p}=0,039$ ), existiendo mejor escolaridad (secundaria o universitaria) entre las madres de adolescentes control que entre los casos $(52,6 \%$ vs $37,8 \%$; $\mathrm{p}<0,001)$. De otro lado, hubo mayor educación en temas de sexualidad por parte de las madres hacia sus hijas entre las adolescentes control $(74,8 \%$ vs $62,9 \% ; p<0,001)$, sin diferencias entre los dos grupos en temas de educación en planificación familiar por parte de la madre a sus hijas. El 16,6\% y $37,4 \%$ de las madres de adolescentes casos y control respectivamente, conseguían los métodos de planificación familiar respectivamente $(p<0,001)$. Por el lado de los padres de las adolescentes, encontramos igualmente mejor escolaridad (secundaria y universitaria) entre los padres de las adolescentes control que entre los casos $(62,9 \%$ vs $57 \%$; $\mathrm{p}<0,001)$. Un número menor de padres hablan sobre métodos de planificación familiar entre las adolescentes caso que entre las adolescentes control, (13,9\% vs 25,2\%; p<0,016) (Tabla III).

En aspectos socio-culturales, encontramos que la mayor parte de la adolescentes caso pertenecían al estrato socioeconómico 1 (63,6\% vs $28,5 \%$; $\mathrm{p}<0,001)$, no habían recibido educación sexual (20,5\% vs $6,3 \%$; $p<0,001)$, ni educación en planificación familiar $(23,8 \%$ vs $5,6 \% ; p<0,001)$ por parte de docentes en colegios o por personal de salud. De otro lado el $92,1 \%$ de las adolescentes caso y $97 \%$ de adolescentes control, los amigos o pares aprobaban o tenían permisividad hacia la actividad sexual en la adolescencia, $(p=0,016)$ (Tabla IV).

No hubo diferencia significativa entre los dos grupos de adolescentes para edad de la menarca, aprobación y permisividad de los padres hacia la actividad sexual en la adolescencia y si la hija era única o no. Tampoco hubo diferencia significativa para edad del hermano mayor, edad materna, educación de la madre a su hija en temas de planificación familiar, edad del padre, ocupación del padre, educación sexual hacia la adolescente por el padre y edad en que la mamá de la adolescente fue madre (Tablas I, II y III).

Entre las variables individuales hallamos asociación entre embarazo en mujeres adolescente y realización de otras actividades diferentes a estudiar (OR: 2,5; IC95\%: 1,7-3,9), escolaridad de 9 o menos años aprobados (OR: 3,1; IC95\%: 2-4,7), inicio de la actividad sexual a los 15 o menos años de edad (OR: 2,2; IC95\%: 1,4-3,5), no usar métodos de planificación familiar en todas las relaciones sexuales (OR: 3,1; IC95\%: 2-5,1) y no uso de condón masculino por parte de las parejas sexuales de las adolescentes en todas las relaciones sexuales (OR: 3,2; IC95\%: 1,9-5,7) (Tabla V). 
Tabla III

CARACTERÍSTICAS FAMILIARES

Variable familiar
Vive o no con los padres
Con quién vive la adolescentes en el momento del embarazo
Cónyu
Número de personas con quien habita la adolescente
Padres de la adolecente aprueban o desaprueban las RS
Lámero adolescente es hija única

Lugar que ocupa la adolecente entre sus hermanos

Edad en años de hermano mayor de la adolescente

Control

No conoce la mamá

Edad en años de la madre de la adolescente

Ocupación de la madre de la adolescente

$$
\text { Empleada o trabajadora independiente }
$$

Ama de casa

Escolaridad de la madre de la adolescente

Ninguna
Primaria
Secundaria
Universidad

Educación sexual por la madre a la adolescente

Universidad

$$
\text { Si }
$$

Madre de la adolescente le habla sobre PF

No

Madre de la adolescente le consigue métodos de PF

Padre de la adolescente vive

$$
\begin{array}{r}
\text { Si } \\
\text { No } \\
\text { No conoce al papá }
\end{array}
$$

Edad en años del padre de la adolescente

Ocupación del padre de la adolescente

$$
\text { Empleado o trabajador independiente }
$$
Desempleado o jubilado
$291(94,6 \%)$

$11(5,4 \%)$ (n: 302)

$249(82,5 \%)$

n: 53

$12(22,6 \%)$

$5(9,4 \%)$

$12(22,6 \%)$

$19(35,8 \%)$

$5(9,4 \%)$

4 (RI: 3-5)

$115(38,1 \%)$

$22(7,3 \%)$

2 (RI: 1-3)

$$
\begin{array}{r}
98(32,5 \%) \\
95(31,5 \%) \\
109(36 \%) \\
23,8( \pm 5,6)
\end{array}
$$

$295(97,7 \%)$

$7(2,3 \%)$

$\begin{array}{ll}\text { Caso } & \text { Valor } p \\ \text { (n: 151) }\end{array}$

$$
\begin{array}{rr}
72(47,7 \%) & <0,001 \\
\text { n: } 79 & \\
66(83,5 \%) & \\
1(1,3 \%) & \\
3(3,8 \%) & \\
3(3,8 \%) & \\
6(7,6 \%) & \\
3(\mathrm{Rl}: 2-5) & <0,01 \\
44(29,1 \%) & 0,163 \\
7(4,6 \%) & 0,379 \\
2 \text { (RI: } 1-4) & 0,01
\end{array}
$$

$42(27,8 \%)$

$39(25,8 \%)$

$70(46,4 \%)$

$$
\begin{array}{r}
24,5( \pm 6,4) \quad 0,3413 \\
0,688
\end{array}
$$

$147(97,3 \%)$

$2(1,3 \%)$

$2(1,3 \%)$

$41,4( \pm 6,4)$

$41,4( \pm 7,1)$

0,9354

0,039

$133(44 \%)$

$169(56 \%)$

$51(33,8 \%)$

$100(66,2 \%)$

$<0,001$

$18(11,9 \%)$

$76(50,3 \%)$

$38(25,2 \%)$

$19(12,6 \%)$

$145(48 \%)$

$14(4,6 \%)$

$<0,01$

$95(62,9 \%)$

$56(37,1 \%)$

$76(25,2 \%)$

$106(70,2 \%)$

0,128

$<0,001$

$25(16,6 \%)$

$126(83,4 \%)$

$113(37,4 \%)$

$261(86,4 \%)$

$20(6,6 \%)$

$21(7 \%)$

$123(81,5 \%)$

24 (15,9\%)

$4(2,6 \%)$

$45,9( \pm 8,3)$

$45,6( \pm 7,9)$

0,6833

0,418
$140(92,7 \%)$

$11(7,3 \%)$ 
Continuación. Tabla III

\begin{tabular}{|c|c|c|c|}
\hline Variable familiar & $\begin{array}{l}\text { Control } \\
(n: 302)\end{array}$ & $\begin{array}{c}\text { Caso } \\
\text { (n: 151) }\end{array}$ & Valor $\mathrm{p}$ \\
\hline $\begin{array}{r}\text { Ninguna } \\
\text { Primaria } \\
\text { Secundaria } \\
\text { Universidad }\end{array}$ & $\begin{array}{r}3(1 \%) \\
109(36,1 \%) \\
170(56,6 \%) \\
20(6,3 \%)\end{array}$ & $\begin{array}{l}17(11,3 \%) \\
48(31,8 \%) \\
60(39,7 \%) \\
26(17,2 \%)\end{array}$ & $<0,001$ \\
\hline Educación sexual por el padre a la adolescente & $77(25,5 \%)$ & $33(21,9 \%)$ & 0,492 \\
\hline Padre de la adolescente le habla sobre PF & $76(25,2 \%)$ & $21(13,9 \%)$ & 0,016 \\
\hline Padre de la adolescente le consigue métodos de PF & $17(5,6 \%)$ & $8(5,3 \%)$ & 0,982 \\
\hline Edad en que la mamá tuvo primer hijo o embarazo & 20 (RI: 17-23) & 19 (RI: 17-21) & 0,1204 \\
\hline
\end{tabular}

RS: Relaciones sexuales. PF: Planificación familiar.

Tabla IV

CARACTERÍSTICAS SOCIO-CULTURALES

\begin{tabular}{|c|c|c|c|}
\hline Variable socio-cultural & $\begin{array}{l}\text { Control } \\
(n: 302)\end{array}$ & $\begin{array}{l}\text { Caso } \\
(\mathrm{n}: 151)\end{array}$ & Valor $p$ \\
\hline Estrato socioeconómico & 2 (RI: 1-2) & 1 (RI: $1-2)$ & $<0,001$ \\
\hline Estrato socioeconómico 1 & $86(28,5 \%)$ & $96(63,6 \%)$ & $<0,001$ \\
\hline Recibió educación sexual por personas diferentes de los padres & $283(93,7 \%)$ & $120(79,5 \%)$ & $<0,001$ \\
\hline $\begin{array}{l}\text { No haber recibido educación sexual por parte de personal docente, } \\
\text { de salud u otro diferente a los padres }\end{array}$ & $19(6,3 \%)$ & $31(20,5 \%)$ & $<0,001$ \\
\hline $\begin{array}{l}\text { No haber recibido educación en PF por parte de personal docente, } \\
\text { de salud u otro diferente a los padres }\end{array}$ & $17(5,6 \%)$ & $36(23,8 \%)$ & $<0,001$ \\
\hline $\begin{array}{l}\text { Aprobación o permisibilidad por amigos de la adolecente hacia la } \\
\text { actividad sexual en la adolescencia }\end{array}$ & $293(97 \%)$ & $139(92,1 \%)$ & 0,016 \\
\hline
\end{tabular}

Tabla V

ANÁLISIS BIVARIADO Y MULTIVARIADO DE LAS VARIABLES INDIVIDUALES

\begin{tabular}{|c|c|c|c|c|c|c|}
\hline Variable individual & $\begin{array}{l}\text { Control } \\
(\mathrm{n}: 302)\end{array}$ & $\begin{array}{l}\text { Caso } \\
(n: 151)\end{array}$ & $\begin{array}{c}\text { ORc } \\
(\text { IC } 95 \%)\end{array}$ & Valor $\mathrm{p}$ & $\begin{array}{c}\text { ORa } \\
(\text { IC 95\%) }\end{array}$ & Valor $p$ \\
\hline \multicolumn{7}{|l|}{ Ocupación de la adolescente } \\
\hline & 195 & 63 & & & & \\
\hline Estudia & $(64,7 \%)$ & $(41,7 \%)$ & 1 & $<0,001$ & 1 & $<0,001$ \\
\hline $\begin{array}{r}\text { Otra actividad (trabaja, } \\
\text { ama de casa, otra) }\end{array}$ & $\begin{array}{c}107 \\
(35,3 \%)\end{array}$ & $\begin{array}{c}88 \\
(58,3 \%)\end{array}$ & $\begin{array}{c}2,5 \\
(1,7-3,9)\end{array}$ & & $\begin{array}{c}8,6 \\
(4,4-17,8)\end{array}$ & \\
\hline \multirow{2}{*}{$\begin{array}{l}\text { Escolaridad de } 9 \text { o menos años } \\
\text { aprobados }\end{array}$} & $\begin{array}{c}105 \\
(34,8 \%)\end{array}$ & $\begin{array}{c}94 \\
(62,3 \%)\end{array}$ & $\begin{array}{c}3,1 \\
(2-4,7)\end{array}$ & $<0,001$ & $\begin{array}{c}0,9 \\
(0,5-1,8)\end{array}$ & 0,834 \\
\hline & 188 & 118 & 2,2 & & 2 & \\
\hline \multirow[t]{2}{*}{ Sexarca a los 15 años o menos } & $(62,3 \%)$ & $(78,2 \%)$ & $(1,4-3,5)$ & $<0,001$ & $(1-3,9)$ & 0,049 \\
\hline & $\begin{array}{c}164 \\
(54,3 \%)\end{array}$ & $\begin{array}{c}119 \\
(78,8 \%)\end{array}$ & $\begin{array}{c}3,1 \\
(2-5,1)\end{array}$ & $<0,001$ & $\begin{array}{c}2,4 \\
(1,04-5,6)\end{array}$ & 0,041 \\
\hline $\begin{array}{l}\text { No usar métodos de PF en todas las } \\
\text { relaciones sexuales }\end{array}$ & 199 & 130 & 3,2 & & 1,4 & \\
\hline $\begin{array}{l}\text { No usar condón en todas las relacio- } \\
\text { nes sexuales }\end{array}$ & $(65,9 \%)$ & $(86,1 \%)$ & $(1,9-5,7)$ & $<0,001$ & $(0,6-3,5)$ & 0,483 \\
\hline
\end{tabular}

ORc: Odds ratio crudo. ORa: Odds ratio ajustado. PF: Planificación familiar. 
Las variables familiares asociadas a embarazo en mujeres adolescentes fueron, tener tres o más hermanos (OR: 3,2; IC95\%: 2-5,2), hermano/a que en la adolescencia haya sido padre o madre (OR: 1,6; IC95\%: 1,04-2,4), madre de adolescente ama de casa (OR: 1,5; IC95\%: 1,01-2,4), escolaridad materna menor a 11 años de estudios aprobados (OR: 1,8; IC95\%: 1,2-2,8), no recibir educación sexual por la madre (OR: 1,8; IC95\%: 1,1-2,7), no suministro de métodos de planificación familiar por parte de la madre (OR: 3,0; IC95\%: 1,8-5,1) y la no educación en temas de planificación familiar por parte del padre (OR: 2,1; IC95\%: 1,2-3,7) (Tabla VI).

Las variables socio-culturales asociadas a embarazo en mujeres adolescentes fueron, pertenecer al estrato socio-económico 1 (OR: 4,4; IC95\%: 2,8$6,8)$, no haber recibido educación sexual por parte de personal docente, de salud u otro diferente a los padres (OR: 3,8; IC95\%: 2-7,5), no haber recibido educación en PF por parte de personal docente, de salud u otro diferente a los padres (OR: 5,2; IC95\%:
2,7-10,3) y pares o amigos de la adolecente que aprueban o son permisivos con el inicio de la actividad sexual en la adolescencia (OR: 0,4; IC95\%: $0,1-0,9)$ (Tabla VII).

Después del análisis multivariado, las variables que permanecieron asociadas a embarazo en mujeres adolescentes fueron para las variables individuales, realización al momento del embarazo de otras actividades diferentes a estudiar (OR: 8,6; IC95\%: 4,4-17,8), inicio de la actividad sexual a los 15 o menos años (OR: 2,0; IC95\%: 1-3,9) y no usar métodos de planificación familiar en todas las relaciones sexuales (OR: 2,4; IC95\%: 1,04-5,6) (Tabla $V$ ). Para las variables familiares, la escolaridad materna menor a 11 años de estudio aprobados (OR: 2,8; IC95\%: 1,1-6,9), no educación sexual de la adolescente por la madre (OR: 2,5; IC95\%: 1,01$6,3)$ y no suministro de métodos de planificación familiar por parte de la madre (OR: 5,3; IC95\%: 1,717) (Tabla VI). Para las variables socio-culturales pertenecer al estrato socio-económico 1 (OR: 3,7;

Tabla VI

ANÁLISIS BIVARIADO Y MULTIVARIADO DE LAS VARIABLES FAMILIARES

\begin{tabular}{|c|c|c|c|c|c|c|}
\hline Variable Familiar & $\begin{array}{l}\text { Control } \\
\text { (n: } 302)\end{array}$ & $\begin{array}{l}\text { Caso } \\
(\mathrm{n}: 151)\end{array}$ & $\begin{array}{c}\text { ORc } \\
(\text { IC 95\%) }\end{array}$ & Valor $p$ & $\begin{array}{c}\text { ORa } \\
(\text { IC } 95 \%)\end{array}$ & Valor $p$ \\
\hline Tener tres o más hermanos & $\begin{array}{c}49 \\
(16,2 \%)\end{array}$ & $\begin{array}{c}58 \\
(34,8 \%)\end{array}$ & $\begin{array}{c}3,2 \\
(2-5,2)\end{array}$ & $<0,001$ & $\begin{array}{c}1,6 \\
(0,7-4)\end{array}$ & 0,278 \\
\hline $\begin{array}{l}\text { Hermano (a) que en la adolescente } \\
\text { haya sido padre o madre }\end{array}$ & $\begin{array}{c}97 \\
(32,1 \%)\end{array}$ & $\begin{array}{c}65 \\
(43 \%)\end{array}$ & $\begin{array}{c}1,6 \\
(1,04-2,4)\end{array}$ & 0,022 & $\begin{array}{c}0,5 \\
(0,2-1,4)\end{array}$ & 0,193 \\
\hline Ocupación de la madre & 133 & 51 & & & & \\
\hline $\begin{array}{l}\text { Empleada o trabajadora independiente } \\
\qquad \text { Ama de casa }\end{array}$ & $\begin{array}{c}(44 \%) \\
169 \\
(56 \%)\end{array}$ & $\begin{array}{c}(33,8 \%) \\
100 \\
(66,2 \%)\end{array}$ & $\begin{array}{c}1 \\
1,5 \\
(1,01-2,4)\end{array}$ & 0,036 & $\begin{array}{c}1 \\
1,7 \\
(0,7-3,9)\end{array}$ & 0,219 \\
\hline $\begin{array}{l}\text { Escolaridad materna menor a } 11 \text { años } \\
\text { aprobados }\end{array}$ & $\begin{array}{c}143 \\
(47,4 \%)\end{array}$ & $\begin{array}{c}94 \\
(62,3 \%)\end{array}$ & $\begin{array}{c}1,8 \\
(1,2-2,8)\end{array}$ & 0,003 & $\begin{array}{c}2,8 \\
(1,1-6,9)\end{array}$ & 0,024 \\
\hline $\begin{array}{l}\text { Educación sexual del adolescente por } \\
\text { la madre } \\
\qquad \begin{array}{l}\mathrm{Si} \\
\text { No }\end{array}\end{array}$ & $\begin{array}{c}226 \\
(74,8 \%) \\
76 \\
(25,2 \%)\end{array}$ & $\begin{array}{c}95 \\
(62,9 \%) \\
56 \\
(37,1 \%)\end{array}$ & $\begin{array}{c}1 \\
1,8 \\
(1,1-2,7)\end{array}$ & 0,009 & $\begin{array}{c}1 \\
2,5 \\
(1,01-6,3)\end{array}$ & 0,047 \\
\hline $\begin{array}{l}\text { Suministro de métodos de PF por la } \\
\text { madre de la adolescentes } \\
\qquad \text { Si } \\
\text { No }\end{array}$ & $\begin{array}{c}113 \\
(37,4 \%) \\
189 \\
(62,6 \%)\end{array}$ & $\begin{array}{c}25 \\
(16,6 \%) \\
126 \\
(83,4 \%)\end{array}$ & $\begin{array}{c}1 \\
3 \\
(1,8-5,1)\end{array}$ & $<0,001$ & $\begin{array}{c}1 \\
5,3 \\
(1,7-17)\end{array}$ & 0,005 \\
\hline $\begin{array}{l}\text { Padre no da educación en PF a sus } \\
\text { hijas }\end{array}$ & $\begin{array}{c}226 \\
(74,8 \%)\end{array}$ & $\begin{array}{c}130 \\
(86,1 \%)\end{array}$ & $\begin{array}{c}2,1 \\
(1,2-3,7)\end{array}$ & 0,006 & $\begin{array}{c}0,6 \\
(0,2-1,8)\end{array}$ & 0,401 \\
\hline
\end{tabular}

ORc: Odds ratio crudo. ORa: Odds ratio ajustado. PF: Planificación familiar. 
Tabla VII

ANÁLISIS BIVARIADO Y MULTIVARIADO DE LAS VARIABLES SOCIO-CULTURALES

\begin{tabular}{|c|c|c|c|c|c|c|}
\hline Variable Socio-cultural & $\begin{array}{l}\text { Control } \\
(n: 302)\end{array}$ & $\begin{array}{c}\text { Caso } \\
(n: 151)\end{array}$ & $\begin{array}{c}\text { ORc } \\
\text { (IC 95\%) }\end{array}$ & $\begin{array}{c}\text { Valor } \\
\mathrm{p}\end{array}$ & $\begin{array}{c}\text { ORa } \\
\text { (IC 95\%) }\end{array}$ & $\begin{array}{c}\text { Valor } \\
p\end{array}$ \\
\hline Estrato socioeconómico 1 & $\begin{array}{c}86 \\
(28,5 \%)\end{array}$ & $\begin{array}{c}96 \\
(63,6 \%)\end{array}$ & $\begin{array}{c}4,4 \\
(2,8-6,8)\end{array}$ & $<0,001$ & $\begin{array}{c}3,7 \\
(2,3-5,7)\end{array}$ & $<0,001$ \\
\hline $\begin{array}{l}\text { No haber recibido educación } \\
\text { sexual por parte de personal } \\
\text { docente, de salud u otro dife- } \\
\text { rente a los padres }\end{array}$ & $\begin{array}{c}19 \\
(6,3 \%)\end{array}$ & $\begin{array}{c}31 \\
(20,5 \%)\end{array}$ & $\begin{array}{c}3,8 \\
(2-7,5)\end{array}$ & $<0,001$ & $\begin{array}{c}2 \\
(0,9-4,4)\end{array}$ & 0,080 \\
\hline $\begin{array}{l}\text { No haber recibido educación } \\
\text { en PF por parte de personal } \\
\text { docente, de salud u otro dife- } \\
\text { rente a los padres }\end{array}$ & $\begin{array}{c}17 \\
(5,6 \%)\end{array}$ & $\begin{array}{c}36 \\
(23,8 \%)\end{array}$ & $\begin{array}{c}5,2 \\
(2,7-10,3)\end{array}$ & $<0,001$ & $\begin{array}{c}3,5 \\
(1,6-7,5)\end{array}$ & 0,001 \\
\hline $\begin{array}{l}\text { Pares o amigos de la adolecen- } \\
\text { te aprueban o son permisivos } \\
\text { hacia la actividad sexual en la } \\
\text { adolescencia }\end{array}$ & $\begin{array}{c}293 \\
(97 \%)\end{array}$ & $\begin{array}{c}139 \\
(92,1 \%)\end{array}$ & $\begin{array}{c}0,4 \\
(0,1-0,9)\end{array}$ & 0,018 & $\begin{array}{c}0,6 \\
(0,2-1,6)\end{array}$ & 0,285 \\
\hline
\end{tabular}

ORc: Odds ratio crudo. ORa: Odds ratio ajustado. PF: Planificación familiar.

IC95\%: 2,3-5,7) y no haber recibido educación en PF por parte de personal docente, de salud u otro diferente a los padres (OR: 3,5; IC95\%: 1,6-7,5) (Tabla VII).

Este modelo con las ocho variables, dado los diferentes coeficientes de RL quedó construido con criterios mayores y criterios menores (Tabla VIII). Entre los criterios mayores encontramos desarrollar al momento del embarazo otras actividades diferentes a estudiar, no usar métodos de planificación familiar en todas las relaciones sexuales, no suministro de métodos de planificación familiar por parte de la madre y no haber recibido educación en PF por parte de personal docente, de salud u otro diferente a los padres. Entre los criterios menores quedaron incluidos, inicio de la actividad sexual a los 15 o menos años, escolaridad materna menor a 11 años de estudio aprobados, no educación sexual del adolescente por la madre y pertenecer al estrato socioeconómico 1 (Tabla VIII). La escala se considera positiva si hay tres criterios mayores, o dos mayores y uno menor, o uno mayor y tres menores presentes, con una sensibilidad 90,1\% (IC95\%: 85-95,2\%), especificidad $81,1 \%$ (IC95\%: 76,6-85,7\%), valor predictivo positivo 70,5\% (IC95\%: 63,8-77,2\%), valor predictivo negativo 94,2\% (IC95\%: 91,2-97,3\%), índice de validez 84,1\% (IC95\%: 80,6-87,6\%) y área bajo ROC 0,856 (IC95\%: 0,823-0,889).

\section{DISCUSIÓN}

El presente trabajo nos permitió conocer las características individuales, familiares y sociales de mujeres adolescentes en embarazo o con hijos, y determinar aquellos factores de riesgo para que una adolescente con actividad sexual sea madre o esté embarazada.

Encontramos que 6\% de las adolescentes embarazadas fueron menores de 15 años, es decir, quedando embarazada o siendo madre tres años más tarde de que ha ocurrido en promedio la menarca, y esto está asociado con el inicio precoz de la AS, la cual para las adolescentes embarazadas o con hijos, está iniciando en el 39,1\% antes de los 15 años de edad. El padre del hijo de la adolescente de 14 o menos años, en un $71,4 \%$ es de 20 o más años de edad. El $59 \%$ de los embarazos fueron no planeados, mientras el 7,9\% de las gestantes pensó en algún momento, interrumpir el embarazo. Entre las causas de este embarazo no planeado y justificadas por las mismas adolescentes fueron en $94 \%$ de las veces, no usar métodos de planificación familiar o usar estos de manera inadecuada.

Desde el nivel individual, el no encontrase estudiando o escolarizada, estuvo fuertemente asociado al embarazo en este grupo de adolescentes. Para Colombia en 2010 (14), el 51\% de mujeres 


\section{Tabla VIII}

\section{MODELO PREDICTOR DE EMBARAZO EN MUJERES ADOLESCENTES}

\begin{tabular}{|c|c|c|}
\hline Variables del modelo & $\begin{array}{l}\text { Coeficiente de regresión } \\
\text { (IC 95\%) }\end{array}$ & Valor $\mathrm{p}$ \\
\hline \multicolumn{3}{|l|}{ Criterios mayores } \\
\hline $\begin{array}{r}\text { Desarrollar al momento del embarazo otras } \\
\text { actividades diferentes a estudiar }\end{array}$ & $1,33(0,19-2,47)$ & 0,022 \\
\hline No usar métodos de PF en todas las relaciones sexuales & $1,79(0,69-2,89)$ & 0,001 \\
\hline No suministro de métodos de PF por parte de la madre & $1,51(0,39-2,64)$ & 0,008 \\
\hline $\begin{array}{r}\text { No haber recibido educación en PF por parte de personal } \\
\text { docente, de salud u otro diferente a los padres }\end{array}$ & $1,92(0,71-4,31)$ & 0,001 \\
\hline \multicolumn{3}{|l|}{ Criterios menores } \\
\hline Inicio de la actividad sexual a los 15 o menos años de edad & $0,82(0,25-1,89)$ & 0,03 \\
\hline Escolaridad materna menor a 11 años de estudio aprobados & $0,45(0,23-1,09)$ & 0,04 \\
\hline No educación sexual del adolescente por la madre & $0,75(0,37-1,88)$ & 0,01 \\
\hline Pertenecer al estrato socio-económico 1 & $0,97(0,48-2,05)$ & 0,03 \\
\hline
\end{tabular}

PF: Planificación familiar.

de 15 a 24 años, se encontraban estudiando, 54\% en la zona urbana y $41 \%$ en la zona rural. Entre las que abandonaron los estudios secundarios, el $11,9 \%$ lo hicieron por no poder pagar los estudios, $7,5 \%$ por estar en embarazo, $6,2 \%$ no quiso estudiar y 5,9\% necesitó trabajar para ganar dinero. Otra razón para no terminar los estudios secundarios, es que se casaron (5\%), especialmente en la zona rural. Un aspecto importante para Colombia, es que las mujeres sin escolaridad, tienen la edad de la primera unión marital a los 17,7 años (14).

Otro factor del nivel individual asociado a embarazo fue el inicio precoz de la AS (15 o menos años de edad). Para Colombia, el 13\% de las adolescentes ha iniciado la AS antes de los 15 años. Sumado a ello, se ha encontrado que las mujeres sin ninguna educación inician relaciones sexuales a los 15,8 años de edad (14). Uno de los factores individuales más importantes en la presencia de un embarazo en mujeres adolescentes fue el no empleo o uso irregular e inadecuado de los métodos de anticoncepción. Al momento del embarazo el 19,2\% se encontraba usando algún método de planificación familiar, entre los cuales, el condón masculino era el más empleado. Uno de los problemas con el embarazo en la adolescencia, es la actitud que toman los hombres frente al embarazo en estas adolescentes. Para Beggs (15), los varones al contar sus historias de "lo que pasó", utilizan tres discursos de género para negar la responsabilidad del embarazo: la feminización del control de la natalidad, un discurso del deseo sexual masculino incontrolable, y el amor, dejando la responsabilidad de la preven- ción del embarazo a la adolescente. En Colombia, se describe que el $81 \%$ ha usado un método anticonceptivo alguna vez en su vida, pero sólo el $10 \%$ los usa en su primera relación sexual (16).

En EEUU dos intervenciones han sido eficaces en la reducción de los embarazos entre los adolescentes, como son la abstinencia sexual y el mejor uso de los anticonceptivos, con lo cual se redujo un $38 \%$ el riesgo global de embarazos, con un $86 \%$ de esta caída atribuible al uso de anticonceptivos. Entre los adolescentes de 15 a 17 años, el $77 \%$ de la reducción del riesgo de embarazos, es atribuida al uso de anticonceptivos y el $23 \%$ a la abstinencia sexual (17). Esto ha hecho sospechar que la promoción de la abstinencia sexual, no es la principal estrategia para prevenir los embarazos en los adolescentes (18).

Entre los factores familiares, encontramos que las madres de las adolescentes juegan un papel importante en temas de sexualidad. Las adolescentes en embarazo o con hijos, tuvieron en mayor proporción madres con baja escolaridad, hecho que mostró asociación a su embarazo. La falta de educación en temas de sexualidad también se halló asociado a embarazo en la adolescencia. De otro lado, el hecho que las madres de las adolescentes no suministraran métodos de planificación familiar estuvo asociado a embarazo en la adolescencia.

Entre los factores que han sido descritos asociados con la reducción de las tasas de embarazos en adolescentes, está la educación sexual adecuada, que no sólo debe ser impartida en colegios y por personal de salud, sino que debe darse desde 
los hogares por parte de padres y madres, sumado a la información en temas de SSR dirigidos de manera específica a los adolescentes en los servicios de salud, que como requisitos, además de guardar confidencialidad, deben ser agradables, que vinculen programas de "opciones de vida" para los adolescentes, vinculando a los padres en esta labor, así como la educación familiar y del adolescente en la resolución de problemas y habilidades para la toma de decisiones, mejorando la comunicación sobre el sexo entre adolescentes y padres, y adolescentes y equipos de salud (19).

Entre los factores socio-culturales, las adolescentes de los estratos socioeconómicos 1 fueron las que más se vieron afectadas por embarazo, constituyendo a este estrato en un grupo de riesgo. Al respecto, Alba (20), describe como los adolescentes de familias de bajo nivel educativo y de ingresos, inician la AS más precozmente y tienen una mayor frecuencia de embarazos. Pero a su vez, la pobreza, el bajo nivel educativo y la baja cobertura de servicios de salud, fueron identificados como los principales factores de riesgo para practicar un aborto inducido en Colombia. Santos y cols (21), describen que el $50 \%$ de las adolescentes embarazadas comparado con $28,6 \%$ de adultas son de estrato socio-económico bajo, $63,6 \%$ son de familia no nuclear vs $24,3 \%$ de las adultas y $51,5 \%$ de sus madres tuvieron su primer embarazo en la adolescencia comparado con $35,7 \%$ de adultas. Al encontrarse presentes los tres factores, el riesgo de un embarazo en adolescente se aumenta de manera significativa (OR: 10,49; IC95\%: 3,43-51,00; $p<0,001)$.

De otro lado, la ausencia de educación en planificación familiar por parte de personal docente o de salud, se vio asociado a la presencia de embarazo en la etapa de la adolescencia. Se ha encontrado que la proporción de información a los adolescentes sobre anticoncepción, no da como resultado un aumento de las tasas de AS, ni un inicio más precoz de la edad de la AS, ni un mayor número de parejas sexuales (22). De hecho, si los adolescentes perciben obstáculos para la obtención de anticoncepción y condones, tienen mayor probabilidad de experimentar resultados negativos relacionados con la SSR (23). Dos trabajo en colegios, demostraron un retraso en el inicio de la AS empleando un enfoque de educación sexual más amplio, que incluía educación en anticoncepción $(24,25)$.

Los factores que han contribuido a la falta de uso de métodos de anticoncepción entre adolescentes o su uso inconsistente, incluyen renuencia a reconocer la $A S$, la creencia de que se es inmune a los problemas como el embarazo o las consecuencias que rodean a la AS. Otros factores incluyen la falta de educación y las ideas erróneas en relación con el uso de los anticonceptivos. A pesar de ello, el nivel de conocimiento que tengan los adolescentes sobre el uso de anticonceptivos, no se correlaciona necesariamente con su empleo constante. Algunos adolescentes no usan o retardan el uso de los anticonceptivos por varias razones, entre las que se cuentan la falta de supervisión de los padres, el temor a que los padres se enteren que ya han iniciado la AS, la ambivalencia y la percepción de que el control de la natalidad es peligrosa o causa efectos adversos no deseados, como por ejemplo el aumento del peso corporal (26-30).

El papel del obstetra y el pediatra frente a la AS, la anticoncepción y la prevención del embarazo en la adolescencia son muy importantes. Los obstetras y pediatras deben alentar la abstinencia y proporcionar asesoramiento sobre reducción de riesgos en los comportamientos sexuales. Idealmente, el asesoramiento debe incluir la discusión sobre la prevención de infecciones de transmisión sexual, educación sobre métodos de anticonceptivos y servicios de planificación familiar para los adolescentes sexualmente activos. Esta discusión debe llevarse a cabo necesariamente en el contexto del desarrollo físico y emocional, así como el bienestar social de estos. Cuando los servicios de anticoncepción se proporcionan por el obstetra y el pediatra se deben tener en cuenta políticas como la confidencialidad, que debe ser explicada a las familias de estos adolescentes, previo a la prestación de estos servicios (31).

Cuando se habla de factores asociados al embarazo cabe destacar el inicio cada vez más temprano del ejercicio de la sexualidad en dolescentes. En lo político, debe constatarse la insuficiente educación sexual en el sistema educativo en muchos de los países, la falta de políticas públicas de salud sexual y reproductiva para adolescentes y mujeres jóvenes no unidas, y la no consideración de los derechos sexuales y reproductivos de las adolescentes.

\section{CONCLUSIONES}

El embarazo en la adolescencia es en la actualidad un grave problema de salud pública, que afecta principalmente a la población más vulnerable, que conlleva riesgos sociales, familiares e individuales, no sólo para la madre adolescente, sino para su hijo. La identificación de características de riesgo en los diferentes niveles, destacan el importante papel que puede desempeñar esta escala como medio fiable para sospechar e identificar a los grupos de adolescentes con riesgo de un embarazo. Las políticas que buscan reducir el número de adolescentes embarazadas tienen que hacer mucho más que solamente brindar información sobre los anticonceptivos y el acceso a ellos. Se requieren intervenciones en las escuelas para brindar a las adolescentes educación sexual de calidad, asistencia en el establecimiento de metas en la vida y apoyo para que permanezcan en la escuela y regresen después del parto. 


\section{REFERENCIAS}

1. Bearinger L, Sieving R, Ferguson J, Sharma V. Global perspective on the sexual and reproductive health of adolescents: patterns, prevention, and potential. Lancet 2007;369:1220-31.

2. Lee MC, Suhng LA, Lu TH, Chou MC. Association of parental characteristics with adverse outcomes of adolescent pregnancy. Fam Pract 1998;15:336-42.

3. Klima CS. Centering pregnancy: a model for pregnant adolescents. J Midwifery Womens Health 2003;48:220-5.

4. WHO guidelines on preventing early pregnancy and poor reproductive outcomes among adolescents in developing countries. World Health Organization 2011. Disponible en: http://www.who.int/maternal_ child_adolescent/documents/preventing_early_pregnancy/en/index.html.

5. Committee on Adolescence. Contraception and Adolescents. Pediatrics 2007;120;1135-48.

6. Plan Andino para la prevención del embarazo en adolescentes. Disponible en: http://www.planandinopea. org/?q=node/60.

7. United States Agency for International Development, Profamilia. Salud sexual y reproductiva en zonas marginadas: situación de las mujeres desplazadas 2005. Bogotá: United States Agency International Development, Profamilia; 2005.

8. Plan Andino para la prevención del embarazo en adolescentes. Disponible en: http://www.planandinopea. org/?q=node/61.

9. Plan Andino para la prevención del embarazo en adolescentes. Disponible en: http://www.planandinopea. org/?q=node/58.

10. Plan Andino para la prevención del embarazo en adolescentes. Disponible en: http://www.planandinopea. org/?q=node/59.

11. Tomas A. 2008 Epitools: Epidemiology Tools. R package versión 0.5-0. http://sites.google.com/site/medepi.

12. Tobias S, Oliver S, Niko B, Thomas L. 2007 ROCR: Visualizing the Performance of Scoring Classifiers. R Package Version 1.0-2. http://rocr.bioinf.mpi-sb.mpg. $\mathrm{de} /$.

13. Marôco J. Análise Estatistica com o PASW Statistics, 1 edn. Pêro Pinheiro, Portugal: Marôco, 2010.

14. Profamilia, Ministerio de la Protección Social República de Colombia, Bienestar Familiar y United States Agency International Development, USAID del Pueblo de los estados Unidos. Conocimientos del VIH y otras ETS, Encuesta Nacional de Demografía y salud ENDS 2010. Informe de prensa, 2010. Disponible en: http://www.profamilia.org.co/encuestas/Profamilia/ Profamilia/index.php?option=com_content\&view=arti cle\&id $=62 \&$ temid $=9$.

15. Beggs J. Becoming teen fathers: stories of teen pregnancy, responsibility, and masculinity. Gender \& Society 2012;26:900-921.
16. Profamilia, Bienestar Familiar, United States Agency for International Development, United Nations Population Fund. Salud sexual y reproductiva en Colombia, Encuesta Nacional de Demografía y Salud 2005. Bogotá: Profamilia, Bienestar Familiar, United States Agency International Development, United Nations Population Fund; 2005.

17. Santelli JS, Lindberg LD, Finer LB, Singh S. Explaining recent declines in adolescent pregnancy in the United States: The contribution of abstinence and improved contraceptive use. Am J Public Health 2007; $97: 150-6$.

18. Pinkerton SD. A relative risk-based, disease-specific definition of sexual abstinence failure rates. Health Educ Behav 2001;28:10-20.

19. Tripp J, Viner R. ABC of adolescence sexual health, contraception, and teenage pregnancy. BMJ 2005;330:590-3.

20. Alba LE. Salud de la adolescencia en Colombia: bases para una medicina de prevención. Univ Méd Bogotá (Colombia) 2010;51:29-42.

21. Santos MI, Rosário F. A score for assessing the risk of first-time adolescent pregnancy. Family Practice 2011;28:482-8.

22. Kirby DB, Laris BA, Rolleri LA. Sex and HIV education programs: their impact on sexual behaviors of young people throughout the world. J Adolesc Health 2007;40:206-17.

23. Guttmacher S, Lieberman L, Ward D, Freudenberg N, Radosh A, Des Jarlais D. Condom availability in New York City public high schools: relationships to condom use and sexual behavior. Am J Public Health 1997;87:1427-33.

24. Kirby D, Barth RP, Leland N, Fetro JV. Reducing the risk: impact of a new curriculum on sexual risk-taking. Fam Plann Perspect 1991;23:253-63.

25. Zabin LS, Hirsch MB, Smith EA, Streett R, Hardy JB. Evaluation of a pregnancy prevention program for urban teenagers. Fam Plann Perspect 1986;18:119-26.

26. Abma JC, Martinez GM, Mosher WD, Dawson BS. Teenagers in the United States: sexual activity, contraceptive use, and childbearing, 2002. Vital Health Stat 23. 2004;(24):1-48.

27. Santelli JS, Lindberg LD, Abma J, McNeely CS, Resnick M. Adolescent sexual behavior: estimates and trends from four nationally representative surveys. Fam Plann Perspect 2000;32:156-65, 194.

28. Brooks-Gunn J, Furstenberg FF Jr. Adolescent sexual behavior. Am Psychol 1989;44:249-57.

29. DiClemente RJ, Wingood GM, Crosby R, et al. Parental monitoring: association with adolescents' risk behaviors. Pediatrics 2001;107:1363-68.

30. Moore PJ, Adler NE, Kegeles SM. Adolescents and the contraceptive pill: the impact of beliefs on intentions and use. Obstet Gynecol 1996;88(3 suppl):48S-56S.

31. Reddy DM, Fleming R, Swain C. Effect of mandatory parental notification on adolescent girls' use of sexual health care services. JAMA 2002;288:710-4. 